\title{
Tipos de ceguera y alteraciones posturales en estudiantes con discapacidad visual
}

\author{
Types of blindness and postural disorders in students with visual \\ impairment
}

\author{
Dina de la Torre-Castro ${ }^{1}$ \\ Yaneth Figueroa-Huamani ${ }^{2}$ \\ Katia Huarcaya-Huasasquiche ${ }^{2}$ \\ Sadith Peralta-Gonzales ${ }^{3}$
}

\begin{abstract}
Resumen
Objetivo: determinar la relación entre tipos de ceguera y alteraciones posturales en estudiantes con discapacidad visual de una institución educativa de Lima Norte, Perú. Materiales y métodos: estudio correlacional de corte transversal, tuvo como muestra aleatoria a 96 estudiantes de ambos sexos, entre 8 y 50 años de edad. Se empleó como instrumentos el Posturograma, el cual midió alteraciones posturales como antepulsión de cabeza, hipercifosis e hiperlordosis; y el Índice de Hernández Corvo para determinar alteraciones en huella plantar, entre ellas, pie cavo y pie plano. Por otra parte, la variable tipos de ceguera fue extraída de la historia clínica institucional de cada individuo. Resultados: $72.9 \%$ de la muestra evaluada eran varones, $41.7 \%$ tenía ceguera total, $96.9 \%$ presentaba antepulsión de cabeza, al relacionar tipos de ceguera y alteraciones posturales se obtuvieron valores de $\mathrm{P}$ mayores a 0.05. Conclusión: en la muestra evaluada no existe relación entre tipos de ceguera y alteraciones posturales.
\end{abstract}

Palabras clave: Ceguera; Postura; Pie; Cabeza; Cifosis (Fuente: DeCS).

\begin{abstract}
Objective: to determine the relationship between types of blindness and postural alterations in students with visual impairment of an educational institution in Lima North, Peru. Materials and methods: cross-sectional correlational study, had as random sample of 96 students of both sexes, between 8 and 50 years of age. The Posturogram was used as instruments, which measured postural alterations such as head antepulsion, hypercifosis and hyperlordosis; and the Hernández Corvo Index to determine alterations in plantar footprint, including cavo foot and flat foot. On the other hand, the variable types of blindness was extracted from the institutional clinical history of each individual. Results: $72.9 \%$ of the sample evaluated were male, $41.7 \%$ had total blindness, $96.9 \%$ had a head antepulsion, when relating types of blindness and postural alterations, $\mathrm{P}$ values greater than 0.05 were obtained. Conclusion: in the sample evaluated there is no relationship between types of blindness and postural alterations.
\end{abstract}

Keys words: Blindness; Posture; Foot; Head; Khyposis (Source: DeCS).

Para citar:

De la Torre D, Figueroa Y, Huarcaya K, Peralta S. Tipos de ceguera y alteraciones posturales en estudiantes con discapacidad visual. CASUS. 2019;4(3):162-169.

DOI: $10.35626 /$ casus.3.2019.215

${ }^{1}$ Hospital María Auxiliadora.

${ }^{2}$ Hospital Cayetano Heredia.

${ }^{3}$ Universidad Católica Sedes Sapientiae.

Correo electrónico: dinadlt7@gmail.com
Fecha de recepción: 15-08-19

Fecha de envío a pares: 17-08-19

Fecha de aprobación por pares: 25-11-19

Fecha de aceptación: 28-11-19 


\section{INTRODUCCIÓN}

La encuesta de la Organización Mundial de Salud (OMS) refirió que en el año 2014 se hallaron 285 millones de personas que padecían discapacidad visual o ceguera de las cuales 39 millones eran ciegas, lo que significó un $0.7 \%$ de la población mundial; los 246 millones de personas restantes presentaban ceguera parcial, un $90 \%$ de la carga de discapacidad visual se concentró en los países en desarrollo (1-3). El 82\% que padecía ceguera se presentó en individuos de 50 años a más. Ahora bien, durante el periodo 2013 y 2014 la Comisión de salud y población del Congreso de la República Peruana, declaró que se tenía una población de 160 mil ciegos y unas 600 mil personas que sufrían alguna deficiencia visual (1-3).

La deficiencia visual, junto a otras discapacidades, trastornos del movimiento, posturas inadecuadas, etc., se pueden considerar como factores condicionantes a la presencia de alteraciones posturales (4). En este sentido, la postura es el resultado del equilibrio entre las fuerzas musculares anti gravitatorias y la gravedad (5), es regulada por el sistema nervioso central y requiere la interacción del sistema vestibular, el sistema somatosensorial y el sistema visual $(1,6,7)$.

La visión desempeña un papel importante en la estabilización de la postura ya que brinda información al sistema nervioso central sobre dirección, velocidad de los movimientos y la ubicación de los segmentos del cuerpo en el ambiente (8). En consecuencia, la ausencia total o parcial de visión podría coadyuvar indirectamente a variaciones en la postura, equilibrio y movilidad, generando compensaciones que a largo plazo desencadenarían en alteraciones posturales (5).

Las posturas incorrectas que las personas con déficit visual adoptan a modo de compensación para disminuir la distancia entre el objeto a visualizar se mantienen aún después de la pérdida total de la visión, con desviaciones del eje postural y contracturas. Además, en las personas ciegas de nacimiento la imposibilidad de imitar posturas de los demás puede dar como resultado posiciones inadecuadas $(1,9)$.

Actualmente, los trastornos posturales son cada vez más frecuentes, principalmente en los individuos con algún tipo de ceguera por su percepción distorsionada en la calidad de imagen y entorno, o pérdida total de visión (10). Una investigación refiere información relativa a la antepulsión de cabeza (90\%), hipercifosis (40\%) e hiperlordosis $(60 \%)$ como alteraciones posturales más frecuentes en individuos con ceguera (1).

Los estudios sobre factores asociados y prevalencia o presencia de alteraciones posturales en personas con limitaciones visuales son casi inexistentes en Perú. Por tal motivo, el presente estudio tuvo como principal objetivo determinar si existe relación entre los tipos de ceguera y alteraciones posturales en estudiantes con discapacidad visual de una institución educativa de Lima Norte, Perú. Es así que los hallazgos obtenidos proporcionarán información a los especialistas de diferentes campos de la salud para implementar programas de prevención y promoción enfocados en individuos con algún tipo de ceguera; asimismo complementarán e incrementarán la información teórica preexistente y serán de utilidad como precedente para investigaciones posteriores.

\section{MATERIALES Y MÉTODOS}

El estudio fue de enfoque cuantitativo, alcance correlacional y diseño transversal. Cuya población estuvo conformada por 128 estudiantes entre 8 y 50 años con discapacidad visual pertenecientes a una institución educativa de Lima Norte. La muestra estuvo conformada por 96 estudiantes entre varones y mujeres, la cual se obtuvo empleando la fórmula de tamaño muestral para poblaciones finitas. El muestreo fue probabilístico de tipo aleatorio simple, ya que se realizó un sorteo entre los individuos de la población.

Las alteraciones posturales se definen como la pérdida de la relación normal entre diferentes partes de los segmentos del cuerpo humano. Entre ellas se encuentran: antepulsión de cabeza, cuando la cabeza se mantiene por delante del cuerpo en el plano sagital anatómico; hipercifosis, excesiva acentuación de la convexidad exterior de la columna dorsal; hiperlordosis, la cual conlleva a 
un incremento progresivo de la curva fisiológica normal lumbar; pie plano, disminución del arco longitudinal plantar; pie cavo, aumento excesivo del arco longitudinal plantar $(11,12,13)$. Esta variable es de naturaleza cualitativa, tuvo como instrumentos al posturograma para evaluar antepulsión de cabeza, hipercifosis e hiperlordosis, mientras que para evaluar alteraciones en huella plantar se empleó el Índice de Hernández Corvo. A su vez, fue recategorizada en tres grupos: pie plano (plano), pie cavo (cavo, cavo fuerte, cavo extremo) y ausencia (normal plano, normal, normal cavo). Los instrumentos empleados se basaron en la medida estática y son comúnmente utilizados en la práctica clínica $(14,15,16,17,18)$. Asimismo, para mayor certeza de resultados, se tomaron fotografías para contrastar los resultados en cada ángulo (anterior, lateral y posterior).

Los tipos de ceguera o discapacidad visual hacen referencia a la deficiencia o pérdida de las funciones visuales que limitan las actividades del individuo, tomando como parámetro su capacidad o habilidad real y las restricciones en su desempeño, considerando el uso de ayudas ópticas, adaptaciones personales. Tipos y características: discapacidad visual moderada, cuando el individuo realiza tareas utilizando lentes; discapacidad visual grave, realiza tareas visuales con inexactitud. Requiere tiempo para hacer una tarea, necesita de ayuda como lentes o lupas; discapacidad visual casi total o ceguera casi total, percepción de objetos como bultos o siluetas; ceguera total, percepción de luz que la persona no puede utilizar para la adquisición de ningún conocimiento o información $(19,20,21)$. La información relativa a esta variable de naturaleza cualitativa fue extraída de las historias clínicas pertenecientes a la institución educativa.

Asimismo, se incluyeron las siguientes covariables: edad, peso, talla (variables de naturaleza cuantitativa, tomadas de la historia clínica), sexo, tiempo de ceguera, caídas durante la marcha, uso de aditamento, trabajo, dolor (cabeza y cuello, zona lumbar, planta de los pies, no dolor).

Para el análisis estadístico se utilizó el programa Stata versión 12. Para el análisis descriptivo se calculó la media y desviación estándar de las variables cuantitativas, mientras que las variables cualitativas fueron descritas por frecuencias y porcentajes. En cuanto al análisis inferencial, para determinar la relación entre variables cualitativas se aplicó la prueba estadística de chi cuadrado considerando intervalos de confianza de $95 \%$ y un valor de $\mathrm{p} \leq 0.05$ como estadísticamente significativo.

Durante el desarrollo de la investigación se respetaron los derechos y la libre participación, por lo cual, los participantes tenían la posibilidad de abandonar el estudio si así lo deseaban; la información estuvo protegida mediante un sistema de codificación. Cabe resaltar que no hubo conflicto de interés por parte de las investigadoras.

\section{RESULTADOS}

En la tabla 1 se observa que de los 96 individuos evaluados, $72.9 \%$ eran varones; $63.5 \%$ presentó ceguera congénita; el tipo de ceguera con mayor presencia fue la ceguera total $(41.7 \%)$; la alteración postural más frecuente fue antepulsión de cabeza (96.9\%), seguido de hipercifosis (65.6\%), hiperlordosis (29.2\%), pie plano (4.2\%) y pie cavo $(32.3 \%)$. De la muestra estudiada el $63.5 \%$ presentó ausencia de alteración en huella plantar, $88.5 \%$ refirió no haber tenido caídas durante los últimos 6 meses antes de la toma de datos, $38.5 \%$ empleaba aditamentos, $26 \%$ de la muestra declaró trabajar, mientras que el $10.4 \%$ reportó dolor en la zona lumbar.

Respecto a la asociación entre las variables tipos de ceguera y alteraciones posturales, se obtuvieron valores de $\mathrm{p}$ mayores a 0.05 ; sin embargo, se observó que el $97.5 \%$ de individuos con ceguera total presentaba antepulsión de cabeza. Mientras que el $73.5 \%$ de sujetos con discapacidad visual casi total tenía hipercifosis, $37.5 \%$ de individuos con ceguera total presentaba hiperlordosis, $15.79 \%$ de individuos con discapacidad visual grave tenía pie plano. Finalmente, el $40.0 \%$ de sujetos con ceguera total presentó pie cavo, $78.95 \%$ de sujetos con discapacidad visual casi total presentaba ausencia de alteraciones de huella plantar (ver tabla 2). 
Tabla 1. Descripción de la muestra

\begin{tabular}{|c|c|c|c|}
\hline \multicolumn{2}{|l|}{ Variables } & $\mathbf{n}$ & $(\%)$ \\
\hline \\
\hline \multicolumn{2}{|c|}{ Femenino } & 26 & 27.1 \\
\hline \multicolumn{2}{|c|}{ Masculino } & 70 & 72.9 \\
\hline \multicolumn{4}{|c|}{ Tiempo de ceguera } \\
\hline \multicolumn{2}{|c|}{ Ceguera congénita } & 61 & 63.5 \\
\hline \multicolumn{2}{|c|}{1 a 11 meses } & 9 & 9.4 \\
\hline \multicolumn{2}{|c|}{1 año a 5 años } & 16 & 16.7 \\
\hline \multicolumn{2}{|c|}{6 años a 10 años } & 7 & 7.3 \\
\hline \multicolumn{2}{|c|}{11 años a 15 años } & 1 & 1 \\
\hline \multicolumn{2}{|c|}{18 años a mas } & 2 & 2.1 \\
\hline \multicolumn{4}{|c|}{ Tipos de ceguera } \\
\hline \multicolumn{2}{|c|}{$\begin{array}{l}\text { Discapacidad visual } \\
\text { moderada }\end{array}$} & 18 & 18.8 \\
\hline \multicolumn{2}{|c|}{ Discapacidad visual grave } & 19 & 19.8 \\
\hline \multicolumn{2}{|c|}{ Discapacidad visual casi total } & 19 & 19.8 \\
\hline Ceguera & & 40 & 41.7 \\
\hline \multicolumn{4}{|c|}{ Alteraciones posturales } \\
\hline Antepulsión & No & 3 & Alteraciones posturales \\
\hline de Cabeza & Sí & 93 & 96.9 \\
\hline \multirow[t]{2}{*}{ Hipercifosis } & No & 33 & 34.4 \\
\hline & Sí & 63 & 65.6 \\
\hline \multirow{2}{*}{ Hiperlordosis } & No & 68 & 70.8 \\
\hline & Sí & 28 & 29.2 \\
\hline \multicolumn{4}{|c|}{ Alteraciones en huella plantar } \\
\hline \multicolumn{2}{|c|}{ Ausencia } & 61 & 63.54 \\
\hline \multicolumn{2}{|c|}{ Pie plano } & 4 & 4.17 \\
\hline \multicolumn{2}{|c|}{ Pie cavo } & 31 & 32.3 \\
\hline Caídas durante & la marcha & & \\
\hline No & & 85 & 88.5 \\
\hline Hace $2 n$ & meses & 4 & 4.2 \\
\hline Hace $4 \mathrm{n}$ & meses & 4 & 4.2 \\
\hline Hace $6 n$ & meses & 3 & 3.1 \\
\hline Uso aditamento & & & \\
\hline & No & 59 & 61.5 \\
\hline & Sí & 37 & 38.5 \\
\hline Trabajo & & & \\
\hline & No & 71 & 74 \\
\hline & Sí & 25 & 26 \\
\hline Dolor & & & \\
\hline & No dolor & 76 & 79.2 \\
\hline & Cabeza y cuello & 9 & 9.4 \\
\hline & Zona lumbar & 10 & 10.4 \\
\hline & Planta de los pies & 1 & 1 \\
\hline Edad* & $20.7 \pm 12$ & & \\
\hline Peso* & $56.3 \pm 17$. & & \\
\hline Talla* & $1.6 \pm 0.1$ & & \\
\hline
\end{tabular}

*Media \pm Desviación estándar

En la tabla 3, al analizar los tipos de ceguera y las caídas se obtuvo un $\mathrm{p}=0.075$ estadísticamente no significativo. De la muestra evaluada, 11 individuos refirieron haber tenido alguna caída entre los dos a seis meses previos a la evaluación. En cuanto a tipos de ceguera y el uso de aditamento se obtuvo un $\mathrm{p}=0.00$. El $86.49 \%$ de individuos que si empleaba aditamentos tenía ceguera total. No se encontró relación entre los tipos de ceguera y el desarrollar un trabajo $(\mathrm{p}=0.311)$. Aunque, el $42.1 \%$ de individuos que trabajaba tenía discapacidad casi total o ceguera casi total.

En la tabla 4, al analizar antepulsión de cabeza y sus covariables se obtuvieron valores estadísticamente no significativos: caídas $(\mathrm{p}=0.08)$, uso aditamento $(\mathrm{p}=0.85)$, dolor $(\mathrm{p}=0.51)$. En cuanto a hipercifosis y dolor si se encontró significancia estadística $(\mathrm{p}=0.02)$. Por otro lado, solo 19 sujetos con discapacidad visual refirieron dolor en alguna parte del cuerpo. Al verificar la relación entre hiperlordosis y dolor sí se halló significancia estadística $(\mathrm{p}=0.00)$. Igualmente, existe asociación entre las variables alteraciones de huella plantar y el uso de aditamento $(\mathrm{p}=0.03)$.

\section{DISCUSIÓN}

$\mathrm{Al}$ indagar en la relación entre los tipos de ceguera y alteraciones posturales no se halló significancia estadística, estos resultados concuerdan con otro estudio (1) donde se analizó alteraciones posturales que adoptaban los individuos con discapacidad visual. Sin embargo, en ambos estudios se halló un predominio de antepulsión de cabeza, hipercifosis e hiperlordosis. Por otra parte, en otros estudios (22-24), donde se evaluaron alteraciones posturales en personas con y sin discapacidad visual, se encontraron valores significativos en antepulsión de cabeza en individuos con discapacidad visual. Por su parte, un estudio (25) obtuvo un $\mathrm{p}$ valor estadísticamente significativo (0.004) en la relación entre hipercifosis y tipos de ceguera. Ahora bien, en el presente estudio no se encontró relación entre tipos de ceguera y alteraciones posturales (antepulsión de cabeza, hipercifosis) posiblemente debido al tamaño muestral. No obstante, al observar la postura de los individuos con discapacidad visual se puede apreciar que suelen llevar la cabeza hacia adelante (fenómeno que da inicio con la pérdida gradual de la visión a modo de compensación para disminuir la distancia entre el objeto a visualizar) alterando la alineación entre los planos y ejes de la 
Tabla 2. Relación entre alteraciones posturales y tipos de ceguera

\begin{tabular}{|c|c|c|c|c|c|c|c|c|c|c|c|c|c|}
\hline \multirow{3}{*}{$\begin{array}{l}\text { Tipos de } \\
\text { ceguera }\end{array}$} & \multicolumn{13}{|c|}{$\begin{array}{c}\text { Variables } \\
\text { Alteraciones posturales }\end{array}$} \\
\hline & \multicolumn{3}{|c|}{ Antepulsión de cabeza } & \multicolumn{3}{|c|}{ Hipercifosis } & \multicolumn{3}{|c|}{ Hiperlordosis } & \multicolumn{4}{|c|}{ Alteraciones de huella plantar } \\
\hline & $\begin{array}{c}\text { No } \\
\text { n (\%) }\end{array}$ & $\begin{array}{c}\text { Sí } \\
\text { n (\%) }\end{array}$ & $\mathbf{P}$ & $\begin{array}{c}\text { No } \\
\text { n (\%) }\end{array}$ & $\begin{array}{c}\text { Sí } \\
\text { n (\%) }\end{array}$ & $\mathbf{P}$ & $\begin{array}{c}\text { No } \\
\text { n (\%) }\end{array}$ & $\begin{array}{c}\text { Sí } \\
\text { n (\%) }\end{array}$ & $\mathbf{P}$ & $\begin{array}{c}\text { Pie } \\
\text { plano } \\
\text { n (\%) }\end{array}$ & $\begin{array}{l}\text { Pie cavo } \\
\text { n (\%) }\end{array}$ & $\begin{array}{c}\text { Ausencia } \\
\text { n (\%) }\end{array}$ & $\mathbf{P}$ \\
\hline $\begin{array}{l}\text { Discapacidad } \\
\text { visual } \\
\text { moderada }\end{array}$ & $0(0.0)$ & $18(100)$ & 0.752 & $7(38.9)$ & $11(61.1)$ & 0.814 & $15(83.3)$ & $3(16.6)$ & 0.359 & $0(0.0)$ & $5(27.78)$ & $13(72.22)$ & 0.086 \\
\hline $\begin{array}{l}\text { Discapacidad } \\
\text { visual grave }\end{array}$ & $1(5.3)$ & $18(94.7)$ & & $6(31.6)$ & $13(68.4)$ & & $15(78.9)$ & $4(21.0)$ & & $3(15.79)$ & $6(31.58)$ & $10(52.63)$ & \\
\hline $\begin{array}{l}\text { Discapacidad } \\
\text { visual casi } \\
\text { total o } \\
\text { ceguera casi } \\
\text { total }\end{array}$ & $1(5.3)$ & $18(94.7)$ & & $5(26.3)$ & $14(73.7)$ & & $13(68.4)$ & $6(31.5)$ & & $0(0.0)$ & $4(21.05)$ & $15(78.95)$ & \\
\hline Ceguera total & $1(2.5)$ & $39(97.5)$ & & $15(37.5)$ & $25(62.5)$ & & $25(62.5)$ & $15(37.5)$ & & $1(2.5)$ & $16(40.0)$ & $23(57.5)$ & \\
\hline
\end{tabular}

Tabla 3. Relación entre tipos de ceguera y covariables (caídas, uso de aditamentos, trabajo y dolor)

\begin{tabular}{|c|c|c|c|c|c|c|}
\hline \multicolumn{7}{|c|}{ Tipos de ceguera } \\
\hline \multicolumn{2}{|l|}{ Covariables } & $\begin{array}{c}\text { Discapacid } \\
\text { ad visual } \\
\text { Moderada } \\
\text { n }(\%) \\
\end{array}$ & $\begin{array}{l}\text { Discapacid } \\
\text { ad visual } \\
\text { grave } \\
\text { n }(\%) \\
\end{array}$ & $\begin{array}{c}\text { Discapacidad } \\
\text { visual casi total o } \\
\text { Ceguera casi total } \\
\text { n }(\%)\end{array}$ & $\begin{array}{c}\text { Ceguera } \\
\text { total } \\
\text { n }(\%) \\
\end{array}$ & $\mathbf{P}$ \\
\hline \multicolumn{7}{|l|}{ Caídas } \\
\hline & No & $17(20.0)$ & $16(18.82)$ & $17(20.0)$ & $35(41.18)$ & \multirow{4}{*}{0.075} \\
\hline & Hace 2 meses & $0(0.0)$ & $3(75.0)$ & $1(25.0)$ & $0(0.0)$ & \\
\hline & Hace 4 meses & $0(0.0)$ & $0(0.0)$ & $0(0.0)$ & $4(100.0)$ & \\
\hline & Hace 6 meses & $1(33.33)$ & $0(0.0)$ & $1(33.33)$ & $1(33.33)$ & \\
\hline \multicolumn{7}{|c|}{ Uso de aditamento } \\
\hline & No & $17(28.81)$ & $17(28.81)$ & $17(28.81)$ & $8(13.56)$ & \multirow{2}{*}{0.000} \\
\hline & $\mathbf{S i}$ & $1(52.70)$ & $2(5.41)$ & $2(5.41)$ & $32(86.49)$ & \\
\hline \multicolumn{7}{|l|}{ Trabajo } \\
\hline & No & $13(18.31)$ & $15(21.13)$ & $11(15.49)$ & $32(45.07)$ & \multirow{2}{*}{0.311} \\
\hline & $\mathbf{S i}$ & $5(20.0)$ & $4(16.0)$ & $8(32.0)$ & $8(32.0)$ & \\
\hline \multicolumn{7}{|l|}{ Dolor } \\
\hline & No & 15 (19.74) & $16(21.05)$ & $14(18.42)$ & $31(40.79)$ & \multirow{4}{*}{0.560} \\
\hline & Cabeza y cuello & $0(0.0)$ & $2(22.22)$ & $3(33.33)$ & $4(44.44)$ & \\
\hline & Zona lumbar & $2(20.0)$ & $1(10.0)$ & $2(20.0)$ & $5(50.0)$ & \\
\hline & Planta de los pies & $1(100.0)$ & $0(0.0)$ & $0(0.0)$ & $0(0.0)$ & \\
\hline
\end{tabular}

cabeza, además al adoptar posturas inadecuadas estarían generando hipercifosis y tensión de los músculos cervicales y dorsales (22-25).

Ahora bien, la alteración postural con mayor presencia fue antepulsión de cabeza con 96.9\%. Este resultado coincide con otro trabajo (26) que obtuvo un $90 \%$ en antepulsión de cabeza en personas con deficiencia visual. Estos resultados se pueden deber a que las personas con discapacidad visual leve o moderada, al intentar mejorar el ángulo de visión adoptan posturas anormales en cabeza y cuerpo. Cabe resaltar que dichas posturas tienden a hacerse un hábito y permanecer en los individuos que perdieron la visión $(8,26)$.

En cuanto a la hipercifosis en pacientes con discapacidad visual se obtuvo 65.6\%. Este dato que guarda similitud al 60\% de hipercifosis ya registrado (25). La población de ese estudio fue de niños entre 5 y 12 años a diferencia de la presente investigación que tuvo mayor variación en edades (8 a 50 años). Ahora bien, es importante señalar que los individuos con déficit visual al ser privados de los estímulos provenientes de la visión alteran su desenvolvimiento motor y estructural del cuerpo, lo cual podría generar mayor inclinación de cabeza, desnivelamiento de hombros, desvío lateral de columna e hipercifosis dorsal (25). 
Tabla 4. Relación entre alteraciones posturales y covariables (caídas, uso de aditamento, dolor)

\begin{tabular}{|c|c|c|c|c|c|c|c|c|c|c|c|c|c|}
\hline \multirow[b]{3}{*}{ Covariables } & \multicolumn{9}{|c|}{ Alteraciones posturales } & \multirow{2}{*}{\multicolumn{4}{|c|}{ Alteraciones en huella plantar }} \\
\hline & \multicolumn{3}{|c|}{ Antepulsion de cabeza } & \multicolumn{3}{|c|}{ Hipercifosis } & \multicolumn{3}{|c|}{ Hiperlordosis } & & & & \\
\hline & $\begin{array}{c}\text { No } \\
\text { n (\%) }\end{array}$ & $\begin{array}{c}\mathrm{Si} \\
\mathbf{n}(\%)\end{array}$ & $\mathrm{p}$ & $\begin{array}{c}\text { No } \\
\text { n (\%) }\end{array}$ & $\begin{array}{c}\mathrm{Si} \\
\mathbf{n}(\%)\end{array}$ & $\mathrm{p}$ & $\begin{array}{c}\text { No } \\
\text { n (\%) }\end{array}$ & $\begin{array}{c}\mathrm{Si} \\
\mathbf{n}(\%)\end{array}$ & $\mathrm{p}$ & $\begin{array}{c}\text { Pie plano } \\
\text { n }(\%)\end{array}$ & $\begin{array}{c}\text { Pie cavo } \\
\text { n }(\%)\end{array}$ & $\begin{array}{c}\text { Ausencia } \\
\text { n (\%) }\end{array}$ & $\mathrm{p}$ \\
\hline Caídas & & & 0.08 & & & 0.95 & & & 0.15 & & & & 0.06 \\
\hline No & $2(2.35)$ & $83(97.65)$ & & $30(35.29)$ & $55(64.71)$ & & $63(74.12)$ & $22(25.88)$ & & $4(4.71)$ & $25(29.41)$ & $56(65.88)$ & \\
\hline $\begin{array}{l}\text { Hace } 2 \\
\text { meses }\end{array}$ & $1(25.0)$ & $3(75.0)$ & & $1(25.0)$ & $3(75.0)$ & & $2(50.0)$ & $2(50.0)$ & & $0(0.00)$ & $0(0.00)$ & $4(100.0)$ & \\
\hline $\begin{array}{l}\text { Hace } 4 \\
\text { meses }\end{array}$ & $0(0.0)$ & $4(100.0)$ & & $1(25.0)$ & $3(75.0)$ & & $1(25.0)$ & $3(75.0)$ & & $0(0.00)$ & $3(75.0)$ & $1(25.0)$ & \\
\hline $\begin{array}{l}\text { Hace } 6 \\
\text { meses }\end{array}$ & $0(0.0)$ & $3(100.0)$ & & $1(33.33)$ & $2(66.67)$ & & $2(66.67)$ & $1(33.33)$ & & $0(0.00)$ & $3(100.0)$ & $0(0.00)$ & \\
\hline Uso de adita & & & 0.85 & & & 0.85 & & & 0.45 & & & & 0.03 \\
\hline No & $2(3.39)$ & $57(96.61)$ & & $22(37.29)$ & $37(62.71)$ & & $45(76.27)$ & $14(23.73)$ & & $4(6.78)$ & $14(23.73)$ & $41(69.49)$ & \\
\hline $\mathrm{Si}$ & $1(2.70)$ & $36(97.30)$ & & $11(29.73)$ & $26(70.27)$ & & $23(62.16)$ & $14(37.84)$ & & $0(0.00)$ & $17(45.95)$ & $20(54.05)$ & \\
\hline Dolor & & & 0.51 & & & 0.02 & & & 0.00 & & & & 0.10 \\
\hline No & $2(2.63)$ & $74(97.37)$ & & $32(42.11)$ & $44(57.89)$ & & $65(85.53)$ & $11(14.47)$ & & $3(3.95)$ & $22(28.95)$ & $51(67.11)$ & \\
\hline $\begin{array}{l}\text { Cabeza y } \\
\text { cuello }\end{array}$ & $1(11.11)$ & $8(88.89)$ & & 1(11.11) & $8(88.89)$ & & $1(11.11)$ & $8(88.89)$ & & $1(11.11)$ & $6(66.67)$ & $2(22.22)$ & \\
\hline $\begin{array}{l}\text { Zona } \\
\text { Lumbar }\end{array}$ & $0(0.0)$ & $10(100.0)$ & & $0(0.0)$ & $10(100.0)$ & & $1(10.0)$ & $9(90.0)$ & & $0(0.00)$ & $2(20.0)$ & $8(80.0)$ & \\
\hline $\begin{array}{l}\text { Planta del } \\
\text { Pie }\end{array}$ & $0(0.0)$ & $1(100.0)$ & & $0(0.0)$ & $1(100.0)$ & & $1(100.0)$ & $0(0.00)$ & & $0(0.00)$ & $1(100.0)$ & $0(0.00)$ & \\
\hline
\end{tabular}

Por otro lado, en la población estudiada se registró bajo porcentaje de hiperlordosis (29,2\%). Este hallazgo coincide con otro estudio (27) que obtuvo $10 \%$ de hiperlordosis. Ahora bien, el escaso porcentaje obtenido puede deberse a que la sinergia entre antepulsión de cabeza y cifosis dorsal no acentuaron la lordosis lumbar como deformación secundaria; sin embargo, es conocido que cualquier acentuación en la curvatura dorsal puede generar cambios en la curvatura lumbar para mantener el equilibrio de cargas, esta modificación en las curvaturas también se ve influenciada por la anteversión o retroversión de la pelvis, no obstante en el presente estudio no se evaluaron dichas alteraciones.

Existe relación entre los tipos de ceguera y el uso de aditamentos. Aunque este hallazgo no es posible compararlo con otras investigaciones debido a que la variable aditamentos no ha sido considerada por otros estudios hasta el momento. Sin embargo, la literatura hace referencia a que la pérdida de visión generaría disminución en el equilibrio $(7,28)$, razón por la cual individuos con ceguera podrían requerir un aditamento para facilitar su deambulación.

Ahora bien, ya que las personas con ceguera tienden a agudizar otros sistemas sensoriales gracias a la plasticidad cerebral, el uso de aditamentos sería atribuido solo para identificar objetos cercanos y evitar caídas $(7,28)$.

Por otro lado, se encontró relación entre hipercifosis y dolor, sin embargo el $57.89 \%$ de las personas que no presentaban dolor, tenían hipercifosis. Estos datos no se pudieron comparar con otros estudios debido a que no se encontraron antecedentes que indaguen acerca del dolor e hipercifosis en individuos con discapacidad visual. Ahora bien, dado el porcentaje de hipercifosis se esperaba hallar mayor presencia de dolor en individuos con esta alteración debido a las contracturas musculares que pudiese generar; no obstante el resultado hallado pudo deberse a que esta alteración era leve.

Entre las principales limitaciones del estudio, para una adecuada interpretación de los hallazgos, se debe mencionar el sesgo de memoria debido al tipo de diseño. De igual manera, algunas variables como las caídas durante la marcha pudieran estar sometidas al sesgo de memoria. Igualmente, la dimensión de la muestra de estudio pudiera constituir una limitación, no obstante el tipo de muestreo empleado garantiza buena representatividad. 


\section{CONCLUSIONES}

En la muestra estudiada no existe relación entre tipos de ceguera y alteraciones posturales. Pese a ello, las alteraciones posturales más prevalentes en individuos con algún tipo de ceguera son antepulsión de cabeza (96.9\%) e hipercifosis (65.6\%). Se encontró relación entre tipos de ceguera u uso de aditamento. Se sugiere desarrollar estudios de investigación de distinto alcance y diseño de investigación que analicen el grado de dependencia entre alteraciones posturales y discapacidad visual, o que identifiquen los factores asociados a ambas variables para elaborar planes y programas de intervención temprana. Se sugiere incluir el análisis de otras alteraciones posturales como anteversión y retroversión de la pelvis, genu flexo de rodilla ya que guardan relación con la hiperlordosis lumbar.

\section{REFERENCIAS BIBLIOGRÁFICAS}

1. Duarte A, Gomez M, Hernández 5. Miralles R, Miralles I, Puig M. C. Descripción de las desviaciones posturales en adultos con discapacidad visual congénita y adquirida que asisten a capacitación e inserción laboral en el centro educacional Santa Lucía [tesis de Licenciatura]. Santiago de Chile: Universidad Metropolitana de Ciencias de la Educación; 2014.

2. Organización Panamericana de la Salud. Ceguera y discapacidad visual $2014 \quad$ [internet]. Montevideo, Uruguay: OPS; 2014 [citado el 06 de Junio de 2018]. Disponible en: https://www.paho.org/uru/index.p hp?option=com_content $\& v i e w=a r$ ticle\&id=770:cegueradiscapacidad-visual \&Itemid=247

3. Proyecto de Ley $\mathrm{N}^{\circ} 2185-2012$. Perú: Comisión de salud y población. Promoción de la salud ocular y la prevención de la ceguera por glaucoma: 2015

4. Arnaiz P. Deficiencias visuales y psicomotricidad. España: Organización Nacional de Ciegos Españoles;1994. Biomecánica clínica del aparato locomotor. 1st ed. Barcelona: Masson; 1998.

Villacahua A, Vedia A, Tolaba M, Jimenez M, Aceituno V. Identificación de alteraciones posturales en niños de 8 a 12 años de la Escuela Teresa Bustos de Lemoine de la Ciudad de Sucre. Ciencia, Tecnología e innovación. 2016;12(13):763-70.

Godfrey A, Conway R, Meagher D, O’Laighin G. Direct measurement of human movement by accelerometry. Med Eng Phys. 2008;30(10):1364-86.

8. Borges M, Gomes S, Esteves C, Conti V, Lopes N. Avaliação das alterações posturais e retrações musculares na deficiência visual: estudo de caso. Saúde coletiva. 2011;8(49):77-82.

Maciel P. Discapacidad Visual Y Esquema Corporal. Integrando.org. 2017.

10. Parreira R, Grecco L, Oliveira C. Postural control in blind individuals: A systematic review. Gait \& Posture. 2017.

11. Gamboa J, Naranjo M. Incidencia de la cervical en posturas de antepulsión de cabeza en los estudiantes de la carrera de Terapia Física de la Universidad Técnica de Ambato [Tesis de Licenciatura]. Ambato: Universidad Técnica de Ambato; 2017.

12. Gómez M. Prevención de patologías de la columna vertebral. Universidad Politécnica de Madrid; 2015.

13. Muñoz J. Deformidades del pie. An Pediatr Contin. 2006;4(4):251258.

14. Aguilera J, Heredia JR, Peña G, Segarra V. La Evaluación Postural Estática (EPE) Propuesta de valoración. Instituto Internacional de Ciencias del Ejercicio Físico y la Salud. 2015.p.1-11.

15. Fonseca C. La incidencia de las pausas activas en la salud postural dorsal posterior de los trabajadores universitarios 2017-2018 [Tesis de 
Licenciatura]. Riobamba: Univ Nac Chimborazo; 2018.

16. Cáceres Z. Tipificación de la Huella plantar de escolares entre 6 y 8 años de edad de población Urbana del Municipio de Pamplona. Mov Cient. 2014;8(1):44-52.

20. Registro de Servicio Nacional de Rehabilitación (SNR). Normativa para la certificación de personas con discapacidad visual. 2015.

21. Consejo Nacional de Fomento Educativo. Discapacidad visual. Mexico; 2010.

17. Rey H. Influencia del tipo de pie en el rendimiento del atleta de alta competencia Villa Deportiva Nacional 2017 [Tesis de Licenciatura]. Lima: Universidad Nacional Federico Villarreal; 2019.

18. Lara S, Lara A, Zagalaz M Martínez E. Análisis de los diferentes métodos de evaluación de la huella plantar. Nuevas Tendencias en Educación física, deportes y recreación. 2011;19:49-53.

19. Ministerio de Educación. Guía para la atención de estudiantes con discapacidad visual. 2013. de indivíduos portadores de deficiência visual e auditiva. Universidade Vale do Rio Verde, Trés Coracôes. 2015;13(1):45261.

23. Vomiero B, Thomaz C, Delfino M. Alterações posturais em indivíduos com visão normal e subnormal, analisados por meio da fotogrametria. Revista de Trabalhos Acadêmicos da FAM: TCC 2015. 2016;1(1).

24. Machado H, Rezende R, Baraúna M, Tavares R, Gouveia E. Avaliaçao postural de indivíduos portadores de deficiência visual através da biofotogrametria computadorizada. Fisioterapia em Movimento. 2008;21(2):11-20.

25. Pádua M. Avaliação postural de crianças com deficiência visual [dissertation]. São Paulo: University of São Paulo, Faculdade de Medicina; 2011.

22. Lima J, Silva V. Análise postural 26. Do Rego MC, Cronemberger V,
Tavares M. Análise das principais alteraçoes posturais encontradas em portadores de deficiência visual. 2006.

27. Vega L. Beneficios de los ejercicios de Core Stability sobre la postura de estudiantes con baja visión de $7-16$ años que asisten al Aula de atención para personas con discapacidad visual de la ciudad de Milagro. 2016.

28. Nakata H, Yabe K. Automatic postural response systems in individuals with congenital total blindness. Gait \& Posture. 2001;14(1):36-43. 